\title{
Análise experimental do concreto estrutural com adição de resíduos do processo de recauchutagem de pneus
}

\author{
Priscila Marques Correa ${ }^{1 *}$ (1) \\ Ruth Marlene Campomanes Santana ${ }^{1}$ (D)
}

\section{Resumo}

Atualmente tem-se procurado alternativas para a reciclagem da borracha de pneus. Para isso tem-se estudado a incorporação destes resíduos ao concreto, pois o aumento da disposição destes resíduos em lugares inadequados vem se tornando preocupante, causando problemas sociais, econômicos e ambientais. Diante disso nesta pesquisa, estudou-se o comportamento mecânico do concreto com a adição da borracha como substituto parcial da areia em $10 \%$ e $20 \%$. Foram avaliadas as propriedades mecânicas do concreto em relação a resistência à compressão axial, resistência à tração e o módulo de elasticidade aos 28 dias de cura. Os resultados obtidos apontam que há uma redução nas propriedades mecânicas do concreto e uma redução da rigidez do material, tornando-o mais tenaz, indicando que seria um material apropriado para a produção de lajes. Da mesma forma, a boa incorporação do material ao solo pode auxiliar na construção de obras geotécnicas, onde é necessário que o concreto tenha uma maior deformação. Pode-se concluir que o traço aconselhável foi com substituição em 10\%, apresentou uma redução de 10,5\%,16,4\% e 15,6\% na resistência à compressão axial, resistência à tração e o módulo de elasticidade respectivamente.

Palavras-chave: Borracha; Meio ambiente; Resíduos; Construção civil.

\section{Experimental analysis of the structural concrete with the addition of waste from the tire recovery process}

\begin{abstract}
Currently, alternatives have been sought for the recycling of tire rubber. For this purpose, an incorporation of these residues into concrete has been studied, as the increase in the disposal of these residues in inappropriate places has been causing concern, causing social, economic and environmental problems. Therefore, in this research, the mechanical behavior of concrete was studied with the addition of rubber as a partial substitute for sand in $10 \%$ and $20 \%$ where they were evaluated as mechanical properties of concrete in relation to axial compression strength, tensile strength and the modulus of elasticity at 28 days of curing. The results obtained point out that there is a reduction in the mechanical properties of the concrete and a reduction in the stiffness of the material, making it more tenacious, indicating that it would be an ideal material in the production of slabs, as well as its good incorporation in the soil can help in the construction of geotechnical works, where it is necessary that the concrete has a greater deformation. It may be required that the advisable mix be replaced by $10 \%$, present a reduction of $10.5 \%, 16.4 \%$ and $15.6 \%$ in the axial compression strength, tensile strength and the modulus of elasticity respectively.
\end{abstract}

Keywords: Rubber; Environment; Waste; Construction.

\section{Introdução}

O pneu é um componente indispensável ao atual. No Brasil, somente em 2019 foram produzidos 74,9 milhões de unidades, segundo as informações da ANIP funcionamento dos automóveis por ser o ponto de contato com o solo. Desde que foi criado, no século XIX, passou por diversas modificações até chegar a técnica de fabricação

(Associação Nacional da Indústria de Pneumáticos) [1]. Em 2014 o índice de reciclagem de pneus no Brasil foi de 85\%.

\footnotetext{
${ }^{1}$ Laboratório de Materiais Poliméricos, Departamento de Engenharia, Universidade Federal do Rio Grande do Sul - UFRGS, Porto Alegre, RS, Brasil.

*Autor correspondente: priengenheira@gmail.com
} 
Existem cerca de 30 empresas que processam pneus no país inteiro. Em 2013, foram cadastrados 1127 pontos de coleta (647 localizados nas cidades com população acima de 100 mil habitantes). A capacidade em todas as unidades é superior a 460 mil toneladas por ano. Os veículos de passeio e caminhões de cargas, são os maiores responsáveis no uso de pneus [2] e consequentemente favorecendo para o grande volume deste material descartado. As empresas de fabricação de pneus são responsáveis para a destinação correta do resíduo, assim reduzindo o impacto ambiental, por ser um produto não biodegradável, além disso, a resolução do CONAMA (Conselho Nacional do Meio Ambiente) $416 / 2009$ determina que para cada venda de pneu novo, o fabricante deverá recolher um usado, assim auxiliando para a redução do descarte inadequado [3].

A construção civil vem em busca de matérias-primas para substituir a areia, que atualmente vem sendo limitada devido aos problemas ocasionados nas encostas dos rios. Com isso é necessário encontrar uma alternativa, como é o caso da borracha de pneus. Assim o presente trabalho tem como objetivo a substituição parcial da areia pela borracha em diferentes teores: $10 \%$ e $20 \%$.

\section{Materiais e métodos}

\subsection{Materiais}

O cimento utilizado foi o CPV-ARI Votoran para a confecção dos corpos de prova, com massa específica igual a $3,09 \mathrm{~g} / \mathrm{cm}^{3}$, conforme informação do fabricante. $\mathrm{O}$ agregado miúdo utilizado foi a areia natural proveniente do rio Guaíba, Porto Alegre - RS e o agregado graúdo foi a brita do tipo 1. Utilizou-se, na composição das misturas de concreto, água potável proveniente da rede de distribuição municipal. O resíduo de borracha (Figura 1), proveniente da recauchutagem de pneus inservíveis, foi cedido pela empresa RENOVADORA - Recuperadora de Pneus, localizada na cidade de Porto Alegre, RS.

\subsection{Caracterização dos agregados}

A caracterização dos agregados baseou-se nos ensaios de granulometria, segundo a NBR NM 248 [4], absorção de água, NBR NM 30 [5] massa específica e massa específica aparente, NBR NM 52 [6], massa unitária, NBR NM 45 [7]. E os resultados destas caracterizações são apresentadas na Tabela 1.

A curva granulométrica da borracha foi obtida após seu peneiramento, sendo utilizado o material que passa por esta. A Figura 2 apresenta, as porcentagens retidas em função da abertura das peneiras.

\subsection{Preparação dos corpos de prova}

O traço utilizado foi de 1: 1,64: 2,83 a/c 0,43 e abatimento em $100 \pm 20 \mathrm{~mm}$, calculado através do método EPUSP/ ITP. O traço consiste na proporção cimento: areia: brita, e a relação água cimento $(\mathrm{a} / \mathrm{c})$ respectivamente, sendo que o teor da borracha foi definida através da porcentagem em relação ao volume. O traço em questão foi definido com o objetivo de obter uma resistência de referência de 40 $\mathrm{MPa}$. Foram confeccionados 30 corpos de prova divididos proporcionalmente em REF 0, BR 10, BR 20, contendo $0 \%$, $10 \%$, e $20 \%$ respectivamente. A moldagem foi realizada de acordo com a NBR 5738 [8], o molde cilíndrico possui dimensão de $10 \times 20 \mathrm{~cm}$ (diâmetro $\times$ altura), foi também respeitado a temperatura de $23^{\circ} \mathrm{C}$ e $99 \%$ U.R para um período de cura de 28 dias.

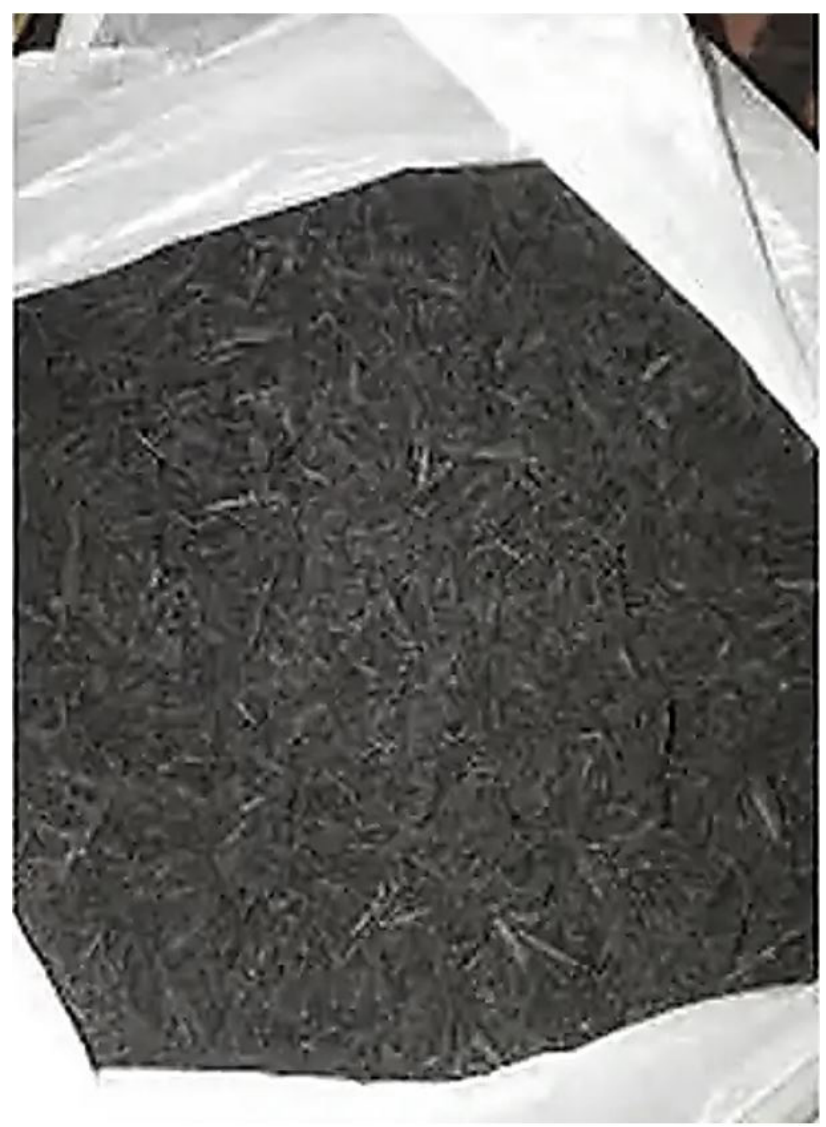

Figura 1. Resíduos de borracha de pneu utilizados.

Tabela 1. Caracterização dos agregados

\begin{tabular}{ccccccc}
\hline & \multirow{2}{*}{ Módulo de finura } & \multicolumn{2}{c}{ Massa especifica $\left(\mathbf{g} / \mathbf{c m}^{\mathbf{3}}\right)$} & \multicolumn{2}{c}{ Massa unitária } & \multicolumn{2}{c}{$\begin{array}{c}\text { Absorção de água } \\
\left(\mathbf{g} / \mathbf{c m}^{\mathbf{3}}\right)\end{array}$} & Aparente & & $(\mathbf{\%})$ \\
\cline { 3 - 6 } & & Seco & 2,654 & 2,651 & 1,53 & 0,29 \\
Areia & 2,53 & 2,871 & 2,982 & 1,515 & 1,41 \\
Brita & 6,39 & &
\end{tabular}




\subsection{Caracterização do concreto}

Os ensaios de resistência a compressão foram realizados de acordo com a norma NBR 5739 [9]. Estes ensaios foram realizados para comparar a resistência à compressão de amostras com e sem adição de resíduos. Também foi avaliada a resistência à tração por compressão diametral e o módulo de elasticidade, seguindo os preceitos das respectivas normas: NBR 7222 [10] e NBR 8522 [11]. Os ensaios foram realizados no Laboratório de Ensaios e Modelos Estruturais (LEME/UFRGS). Inicialmente, os corpos de prova foram instrumentados com dois transdutores de deslocamento do tipo Linear Variable Differential Transformer (LVDT) que é um tipo de indutor eletromagnético, após, todo o conjunto foi posto sob o prato inferior da prensa, modelo Shimadzu UH-I.

\section{Resultados e discussão}

Os corpos de prova foram submetidos a ensaios de resistência à compressão axial, tração por compressão diametral e módulo de elasticidade, todos eles foram submetidos a cargas constantes até o momento de sua ruptura, estando estes de acordo com as normas vigentes para cada ensaio.

\subsection{Ensaio de compressão axial}

Na Figura 3 é apresentado os resultados do ensaio de resistência à compressão axial, onde foi observado que a adição do resíduo proporcionou um decréscimo no valor da resistência à compressão. Mesmo com a ocorrência desta queda considerável do desempenho mecânico de 10,5\% e 21,5\% para as amostras BR10 e BR 20 respectivamente quando comparado com a amostra de referência, foi possível obter um

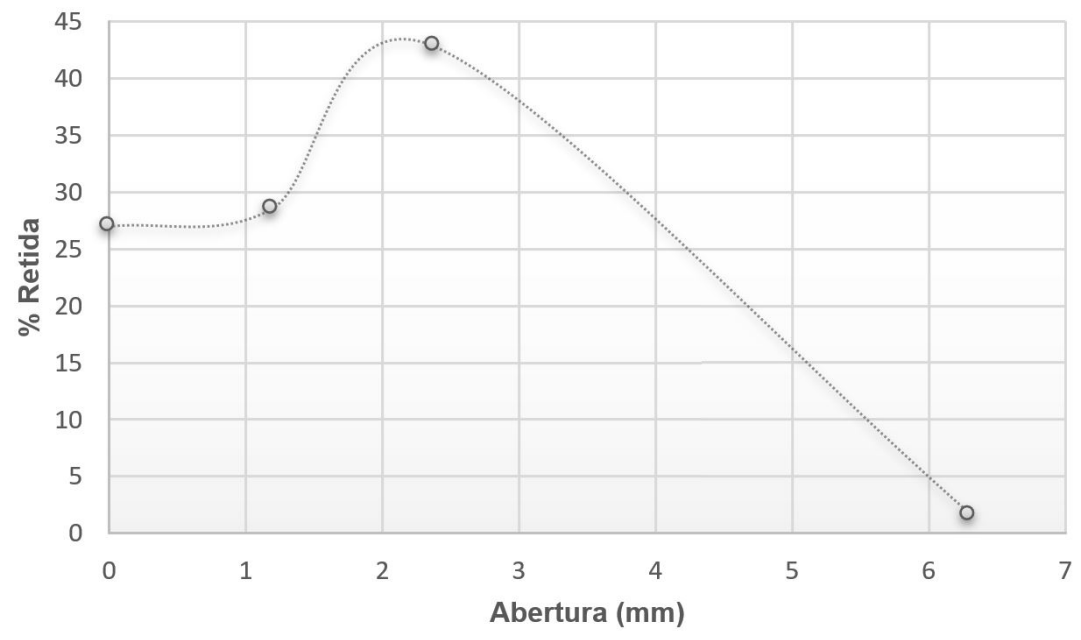

Figura 2. Curva granulométrica de borracha de pneu.
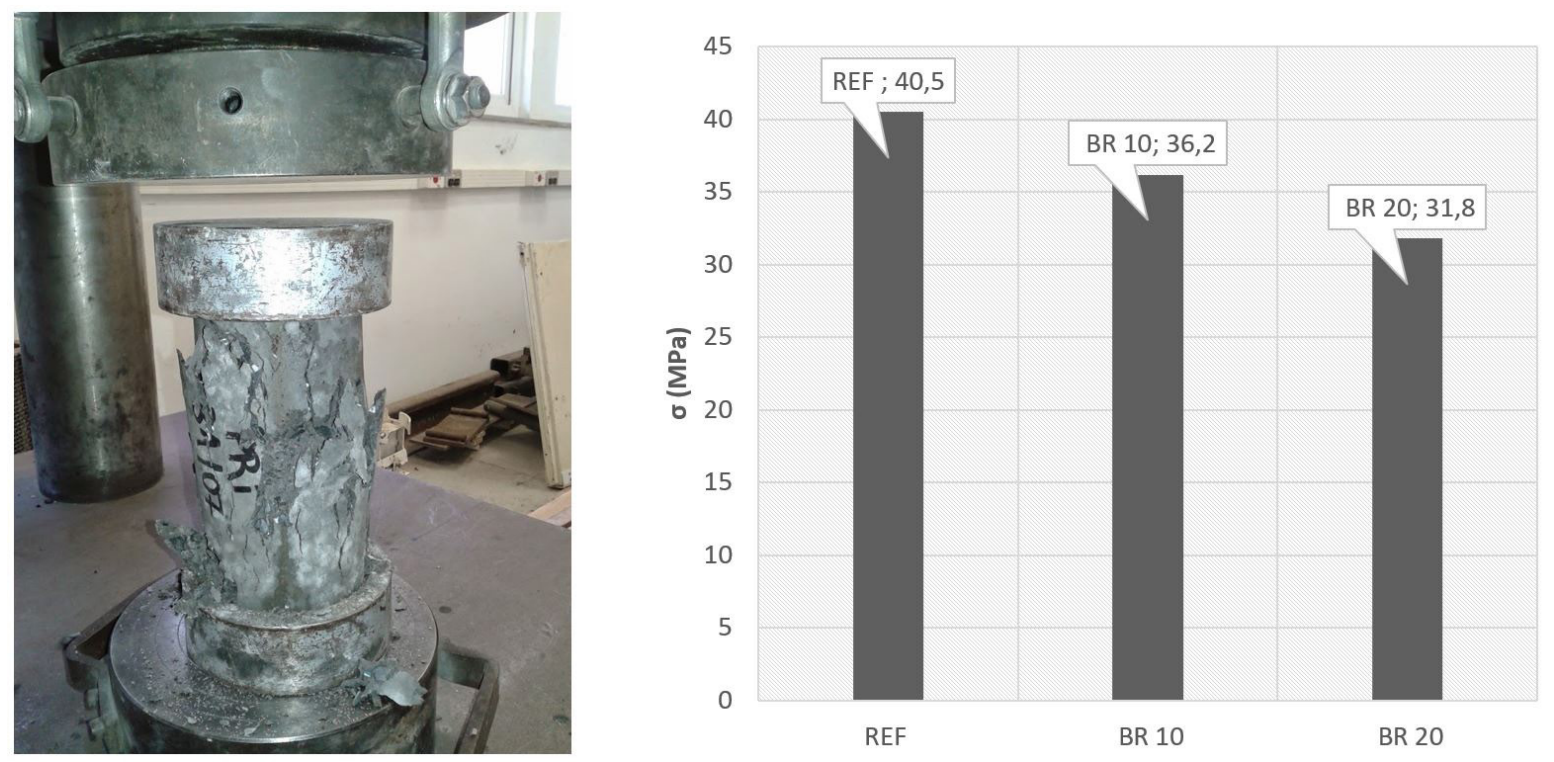

Figura 3. Ensaio de resistência à compressão axial. 

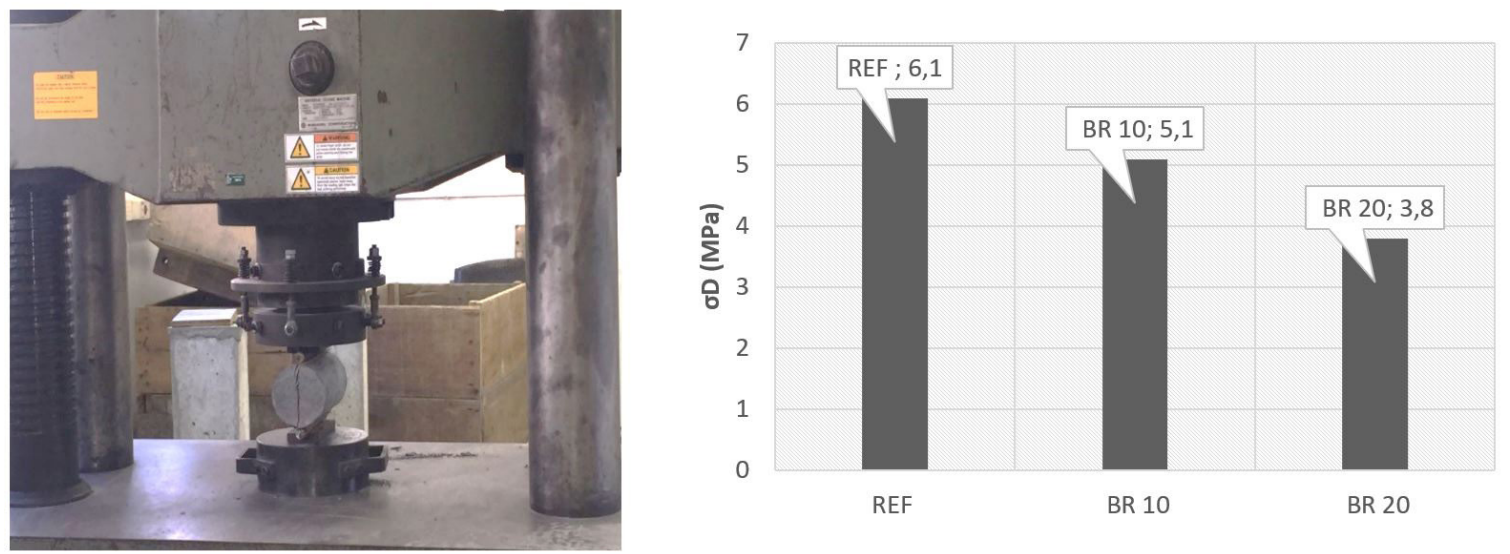

Figura 4. Ensaio de resistência à compressão diametral.
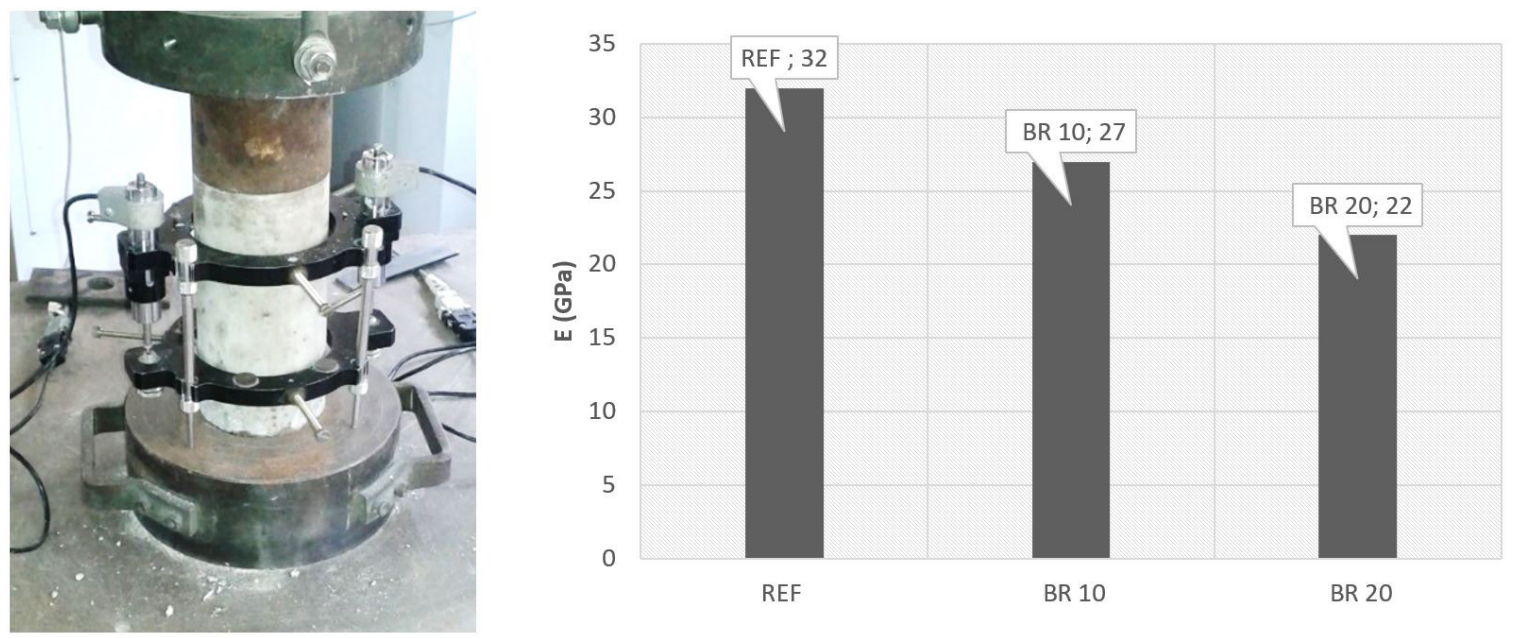

Figura 5. Ensaio de módulo de Deformação Longitudinal.

concreto estrutural de acordo com a classe de agressividade I imposta pela NBR 6118 [12], onde é considerada um risco de deterioração da estrutura como insignificante.

Turatsinze et al. [13] pesquisaram o uso de borracha de pneu em concreto em substituições de $20 \%$, em volume, da areia por borracha de pneu, constataram que após a substituição, a resistência inicial foi reduzida em $50 \%$, segundo os autores isso ocorre devido à baixa aderência entre a borracha e a matriz.

Trigo [2] constatou que aos 28 dias de cura ocorreu uma queda na resistência à compressão, e um aumento no consumo de cimento, na ordem de $15 \%$, não considerando viável a utilização do resíduo.

Embora tenha sido observada uma queda significativa na resistência à compressão, o concreto com a adição de os resíduos de recauchutagem tiveram uma tenacidade aumentada durante os ensaios de compressão axial. Observou-se que antes de romper, o concreto com borracha se deformou significativamente e, quando ocorreu a ruptura, ocorreu menos abruptamente quando comparado ao concreto de referência.

\subsection{Resistência à tração por compressão diametral}

Tecnol Metal Mater Min. 2021;18:e2382
A resistência à tração por compressão diametral e os resultados são apresentados na Figura 4. Após os ensaios as amostras foram comparadas entre si, onde observa-se uma redução na resistência de $16,4 \%$ e 25,5\% para as amostras BR 10 e BR 20 respectivamente quando comparadas a amostra de referência. A diminuição é proporcionalmente maior que a diminuição observada nos resultados da resistência à compressão.

A redução da resistência ocorre devido as características da borracha, por serem mais macias que a areia, outra razão para isso pode ser a ausência de uma coesão adequada entre as partículas da borracha e a massa cimenticia, que por sua vez enfraquece a zona de transição interfacial (ZTI), assim a borracha atua como vazios dentro da matriz cimenticia $[8,12]$ Outras variáveis que influenciam nessa redução: granulometria da borracha, textura da superfície, geometria e propriedades da zona de transição do concreto $[1,14]$.

\subsection{Módulo de deformação longitudinal}

Um ensaio de módulo de elasticidade consiste na aplicação de carga até $30 \%$ da resistência última, com uma 
velocidade de carga controlada de $0,45 \mathrm{MPa} / \mathrm{min}$, no qual se afere os deslocamentos verticais dos CP's com auxílio de LVDTs. Na Figura 5 é apresentado o ensaio em andamento e os resultados obtidos.

Observa-se uma redução $15,6 \%$ e 18,5\% para as amostras de BR 10 E BR 20 respectivamente quando comparado a amostra de referência. A redução é esperada devido a incorporação de um resíduo que apresenta um comportamento elástico (alta capacidade de deformação) e consequentemente favorece na redução da rigidez do concreto, tornando-o mais tenaz [15]. Considerando que o módulo de elasticidade do concreto está diretamente ligado ao da resistência à compressão, uma maior deformação nos concretos contendo borracha significa um módulo de elasticidade menor, o que significa uma queda na resistência à compressão, corroborado pelos resultados obtidos nesta pesquisa.

\section{Conclusões}

\section{Referências}

1 Associação Nacional da Indústria de Pneumáticos. ANIP em números. São Paulo: ANIP; 2019 [acesso em 1 jun. 2020]. Disponivel em: http://www.anip.org.br/anip-em-numeros/dados-gerais/

2 Trigo AM. Estudo de lajes com adição de resíduo de pneu [dissertação]. Ilha Solteira: Universidade Estadual de São Paulo; 2008.

3 Ganjian EH, Khorami M, Maghsoudi AA. Scrap-tyre-rubber replacement for aggregate and filler in concrete. Construction \& Building Materials. 2009;23(5):1828-1836.

4 Associação Brasileira de Normas Técnicas. NBR NM 248/2003: agregados: determinação da composição granulométrica. Rio de Janeiro: ABNT; 2003.

5 Associação Brasileira de Normas Técnicas. NBR NM 30/2001: agregado miúdo: determinação da absorção de água. Rio de Janeiro: ABNT; 2001.

6 Associação Brasileira de Normas Técnicas. NBR NM 52/2009: agregado miúdo: determinação da massa específica e massa específica aparente. Rio de Janeiro: ABNT; 2009.

7 Associação Brasileira de Normas Técnicas. NBR NM 45/2006: agregados: determinação da massa unitária e do volume de vazios. Rio de Janeiro: ABNT; 2009.

8 Associação Brasileira de Normas Técnicas. NBR 5738/2016: procedimento para moldagem e cura de corpos de prova. Rio de Janeiro: ABNT; 2009.

9 Associação Brasileira de Normas Técnicas. NBR 5739/2018: concreto: ensaio de compressão de corpos de prova cilíndrocos. Rio de Janeiro: ABNT; 2018.

10 Associação Brasileira de Normas Técnicas. NBR 7222/2011: concreto e argamassa: determinação da resistência à tração por compressão diametral de corpos de prova cilíndricos. Rio de Janeiro: ABNT; 2011.

11 Associação Brasileira de Normas Técnicas. NBR 8522/2017: determinação dos módulos estáticos de elasticidade e de deformação à compressão. Rio de Janeiro: ABNT; 2017.

12 Associação Brasileira de Normas Técnicas. NBR 6118/2014: projeto de estruturas de concreto: procedimento. Rio de Janeiro: ABNT; 2009.

13 Turatsinze A, Bonnet S, Granju J-L. Mechanical characterisation of cement-based mortar incorporating rubber aggregates from recycled worn tyres. Building and Environment. 2005;40(2):221-226.

14 Marques AC. Estudo da influência da adição de borracha vulcanizada em concreto [dissertação]. Ilha Solteira: Universidade Estadual de São Paulo; 2005. 
Correa et al.

15 Compromisso Empresarial para a Reciclagem. São Paulo: CEMPRE; 2020 [acesso em 1 jun. 2020]. Disponivel em: http://cempre.org.br/artigo-publicacao/ficha-tecnica/id/7/pneus

Recebido em: 5 Jun. 2020

Aceito em: 10 Set. 2020 